\title{
La reforma del Estado y la articulación de los sectores productivos
}

\section{RESUMEN}

La presente investigación tiene tres propósitos. En primer lugar, demostrar la existencia de una débil articulación entre las redes de empresas y el encadenamiento productivo y los sectores que conforman las actividades extractivas y de transformación. En segundo lugar, fundamentar la necesidad de una politica pública de articulación productiva sectorial, de nuevo tipo como condición indispensable para lograr un crecimiento económico sostenido con generación de empleo (inclusión) donde la explotación, producción y transformación estén eslabonadas. En tercer lugar, presentar los lineamientos generales de esta política de fomento de articulación para la productividad y la competitividad del aparato productivo nacional. Finalmente, se hace un planteamiento de un proyecto de Ley y de políticas públicas articuladoras de los sectores productivos que puedan ser implementadas en el mediano o largo plazo y que permita al Estado orientar el desarrollo económico del país de manera articulada a través de sus Sectores Públicos Productivos obligando que en la reforma integral del Estado implique a nivel central, una reducción progresiva de Ministerios e Instituciones Públicas, que a su vez tengan incidencia en la organización más eficiente de los Gobiernos Regionales y Locales, para que estos puedan cumplir su rol de ser los impulsores del desarrollo integral del pais.

Palabras clave: integración de sectores públicos productivos, políticas públicas de articulación productiva

STATE REFORM AND THE ARTICULATION OF THE PRODUCTIVE SECTORS

\section{ABSTRAC}

This research has three purposes. First, prove the existence of weak links between business networks and production chain and the sectors of mining and processing activities. Second, support the need for public policy articulation productive sector, new type as a prerequisite for sustained economic growth through job creation (inclusion) where exploitation, production and processing are crawler's condition. Third, present the broad outlines of this policy of promoting joint to productivity and competitiveness the national productive apparatus. Finally, an approach to a draft law and public policy articulating the productive sectors that can be implemented in the medium or long term and to enable the State to guide economic development of the country in an articulated manner via their public sectors is forcing productive in the comprehensive reform of State involving central level, a progressive reduction of ministries and public institutions, which in turn have an impact on the most efficient organization of regional and local governments, so that they can fulfill their role as the drivers of development of the country.

Keywords: integration of productive public sector, public policies productive articulation

\section{INTRODUCCIÓN}

La presente investigación aporta evidencias y reflexiones sobre la problemática encontrada en la Administración Pública para el trabajo intersectorial en el aspecto de articulación productiva, tanto a nivel Central, Regional y Local. Suministra información y análisis sobre las Políticas Públicas, los

objetivos perseguidos por los diferentes gobiernos y ministerios comprometidos en la materia productiva, asimismo los resultados y logros obtenidos y las dificultades que enfrentan, de forma tal de extraer lecciones para el diseño de modernas Políticas Públicas articuladoras entre los Ministerios de Producción (Industrias y Pesquería), Ministerio de Agricultura y el Ministerio de Energía y Minas, dentro del marco de Reformas de Estado.

En el Marco teórico se pone en evidencia que después del año 2000, hubo algunos esfuerzos importantes, pero éstos han sido puntuales, aislados e insuficientes y que los diferentes gobiernos por los que ha pasado el Perú desde entonces, impulsaron durante la última década, iniciativas públicas que intentaron poner en marcha políticas de apoyo a la actividad productiva, estas experiencias analizadas a lo largo de la investigación muestran claramente la importancia de la puesta en marcha de nuevos procesos de articulación entre instituciones públicas y privadas que definen, cada una con su estilo, velocidad y naturaleza, los nuevos modelos de actuación e interacción en la esfera productiva con impacto en el fortalecimiento institucional.

\section{JUSTIFICACIÓN DE LA INVESTIGACIÓN}

El propósito del estudio es generar reflexión y debate académico sobre el conocimiento existente de la falta de políticas públicas que deben ser generadas por la administración pública, se busca mostrar una solución que permita el desarrollo económico integral del Perú a nivel central, regional y local, la propuesta será de mucha utilidad para los funcionarios públicos que tienen entre sus atribuciones la dación de políticas públicas y modificación de las normas legales que mejoren la organización del Ministerio de la Producción.

\footnotetext{
Ingeniero Industrial, Mag. En Políticas públicas, Profesor asociado de la Facultad de Ingeniería Industrial de la Universidad Nacional del Callao. E-mail: gquintal@hotmail.com

** Licenciado en Ciencias Biológicas, especialista en pesca y acuicultura, jefe del proyecto de conchas de abanico de Chimbote, Universidad Particular Ricardo Palma. E-mail: guillermoqg@gmail.com
} 
Proporcionará elementos de juicio que ayude a la descentralización de la política sectorial de los sectores productivos y se articule en el ámbito central, regional y local, para promover el desarrollo económico y social del Perú.

En la práctica, al proponer cambios en la ley de organización y funciones del Ministerio de la Producción y políticas públicas para el desarrollo integral, ayudará a resolver el problema de la débil articulación de los sectores productivos (extractivos y de transformación) para el desarrollo socio-económico del Perú.

\section{MARCO TEÓRICO}

Antecedentes: Los sectores productivos: son aquellas entidades del Estado cuya función está orientada a promover el desarrollo de la actividad económica del país, estos son los Ministerios: de Agricultura, de Energía y Minas, el Ministerio de la Producción (Vice Ministerio de Pesquería, Vice Ministerio de MYPE e Industria).

\section{a) SECTOR AGRÍCOLA}

El Ministerio de Agricultura (MINAG) identifica la problemática del sector de la siguiente manera:

- Débil articulación de la oferta con el mercado y demanda urbana

- Vulnerabilidad respecto a mercados externos y atraso cambiario

- Deficientes canales de comercialización agrícola

- Escaso capital humano, organizacional e institucional

EI deterioro de los activos agropecuarios: existen pocas tierras cultivables, pero además esto se grava con el proceso de urbanización en las áreas rurales, la erosión de los suelos, entre otros factores.

\section{b) SECTOR MINERO}

El Ministerio de Energía y Minas señala que la producción minera es hoy en día el rubro más importante en las exportaciones del país. Según (KURAMOTO, 2002) la dinámica de estas aglomeraciones o clúster, es la que determina las perspectivas tanto de competitividad de la propia minería como del desarrollo sustentable en este país minero. Las empresas mineras se pueden clasificar en 3 estratos:

- Pequeña minería: aquí se agrupan aquellas empresas cuya producción es menor a 350
TM diarias, y explotan oro y polimetálicos. Generalmente, utilizan técnicas de extracción artesanales y de bajo rendimiento.

- Mediana minería: agrupa alrededor de 30 empresas que operan en unas 70 minas y que producen alrededor del $30 \%$ del total de la minería. explotan al igual que el primer grupo oro y polimetálicos en su mayoría, pero usan métodos de explotación subterráneos y producen concentrados.

- Gran minería: la conforman alrededor de 10 empresas que producen más que 5000 TM diarios. Usan la modalidad de tajo abierto y explotan cobre, hierro, oro y zinc; concentrando alrededor del $55 \%$ de la producción total. Además, son en su mayoría de propiedad extranjera.

\section{c) SECTOR INDUSTRIAL}

El Sub sector Industria es el sector más importante debido al valor agregado que genera en la economía. El Perú es fundamentalmente exportador de materias primas. No obstante, una de las metas trazadas por el gobierno es generar mayores exportaciones en este sector lo cual permite potenciar el desarrollo de industrias con elevado valor agregado y por lo tanto la generación de empleo.

La formación de cadenas productivas dentro del sector manufacturero sería funcional a este objetivo. La política industrial que se aplique al Perú, debiera tratar de impulsar el desarrollo del sector teniendo en cuenta las principales limitaciones o problemas que se encuentren en éste. Se pueden mencionar:

- La baja productividad del trabajo (con una tendencia decreciente en el tiempo), relacionada directamente con la baja calidad de la educación (que afecta la productividad de los trabajadores educados) y la poca inversión e interés empresarial en la capacitación laboral especializada de sus trabajadores.

- Carencia de financiamiento,

- La baja inversión pública en infraestructura, afectando a la estructura de costos de las empresas y limitando su competitividad en los mercados internacionales.

- Deficiente base tecnológica y científica, que impide el mayor desarrollo de las empresas, El mercado interno es muy reducido, no existe una demanda local fuerte y desarrollada que impulse a las empresas a innovar, las exigencias de los clientes se ven satisfechas con los productos 
importados (bienes más baratos que los nacionales y a veces de mejor calidad).

- El tamaño de empresas, que en su mayoría son pequeñas y medianas, restringe las inversiones significativas; la presencia de informalidad que limita el acceso de las empresas al mercado de capitales y perjudica la recaudación tributaria (causado por las imperfecciones es el mercado laboral y una debilidad de los esquemas de derecho de propiedad).

\section{d) SUB SECTOR PESQUERÍA}

Para el caso de este subsector se puede mencionar el mal estado en que se encuentra la atención que el estado le da, desde los recursos de origen hidrobiológico contenidos en las aguas marinas jurisdiccionales, ríos, lagos y otras fuentes hídricas del territorio nacional. A la investigación científica y tecnológica del sector pesquería; así como las condiciones ecológicas de su hábitat; los medios para su conservación y explotación, la calidad, higiene y sanidad de los productos de procedencia acuática; la infraestructura pesquera, así como los servicios adicionales y complementarios para la realización de las actividades extractivas, acuícolas y del proceso pesquero en general, y el tipo de políticas para la promoción de este subsector como país oceánico y el uso de recursos hidrobiológicos para el consumo humano, son completamente desfavorables para el país.

Entonces la pregunta que se deriva de esta aparente situación es:

¿Promoverá la formulación de políticas públicas integrales la articulación de los sectores productivos (extractivos y de transformación) dentro del marco de la reforma del Estado para el desarrollo socioeconómico del país?

Entonces el Objetivo Principal, será el de: Proponer políticas públicas integrales y una nueva ley del Ministerio de la Producción que integre los sectores productivos (extractivos y de transformación) dentro del marco de la reforma del Estado vinculadas al desarrollo nacional, regional y local.

Y los objetivos específicos serán los siguientes:

1. Analizar la articulación institucional de los sectores productivos (extractivos y de transformación) para proponer una nueva ley del Ministerio de Producción.
2. Evaluar la articulación de las actividades productivas empresariales para proponer políticas públicas integrales.

3. Analizar el rol del Estado en el proceso de articulación entre los sectores extractivos y de transformación.

\section{SITUACIÓN PROBLEMÁTICA DE LAS POLÍTI- CAS PÚBLICAS}

Se dice que las políticas públicas constituyen: el vector que sintetiza el conjunto de proposiciones, decisiones y operaciones dinámicas e interdependientes entre actores políticos, sociales e institucionales, a través de las cuales se busca desde el gobierno imprimir un determinado rumbo a la sociedad y el Estado que se gobiernan (MEDELLÍN, 1997).

(OSZLAK Y O'DONNELL, 1976), de otro lado, definen las políticas públicas como: un conjunto de reacciones y omisiones que manifiestan una determinada modalidad de intervención del Estado en relación con una cuestión que concita la atención, interés o movilización de otros sectores de la sociedad civil.

Desde una perspectiva de desarrollo, las políticas, programas e instrumentos de articulación productiva son una buena modalidad de intervención pública con múltiples impactos, ya que no sólo deben atender a los desafíos productivos y competitivos de los aglomerados de empresas, sino que deben además propiciar el fortalecimiento de los factores que vinculan el éxito empresarial con actuaciones más eficientes del sistema de apoyo.

En el presente documento se presentará algunas de las políticas sectoriales aplicadas en los noventa con el objetivo de recordar y apreciar que muchas de ellas quedaron en el papel y que deben ser retomadas por la importancia y trascendencia que tiene a la fecha para el desarrollo integral del país.

Como es sabido, el Perú al igual que muchos países de Latinoamérica en la década del noventa, inició un periodo estabilización económica y de reformas estructurales que comprendieron: un proceso de liberalización y apertura, desregulación de los mercados, reducción del papel del estado, nivelación de los indicadores macroeconómicos: shocks de ajuste, con políticas monetaria y fiscal restrictivas, etc. Predominando la aplicación de políticas horizontales antes que sectoriales. En realidad, las políticas sectoriales se han Ilevado a cabo anteriormente de manera muy limitada, y 
no todos los sectores han sido tratados de igual modo. En el sector industrial por ejemplo se avanzó muy poco en cuanto a este tipo de políticas, mientras que en los sectores primarios se trabajó un poco más al respecto (en particular el sector agropecuario) (Abugattás, 2002).

\section{ANÁLISIS DEL RESULTADO DE LA ARTICU- LACIÓN DE LOS SECTORES PRODUCTIVOS}

Luego de haber identificado a lo largo del marco teórico la situación actual de los sectores extractivos y de transformación en el cual se aprecia la duplicidad de funciones, programas y proyectos, así como la falta de políticas integrales coherentes que articulen el funcionamiento de las actividades productivas (industria, pesquería, agricultura y energía y minas) y hagan más dinámico y coherente el desarrollo integral del país, se viene hasta la fecha manteniendo el Ministerio de la Producción solamente con el sector pesquero.

Si los sectores productivos estuvieran articulados bajo un solo sector que podría ser el Ministerio de la Producción, es desde esta institución que se podría orientar, normar, regular y promover las políticas públicas y la competitividad empresarial dando énfasis en la innovación tecnológica, el aumento de valor agregado y la diversificación productiva; por lo que urge la necesidad de corregir la desarticulación existente de los sectores productivos en el ámbito nacional, regional y local.

De los programas, proyectos y actividades de desarrollo que se pueden apreciar por las informaciones de los Planes Estratégicos de los diferentes sectores existe una fuerte tendencia a la superposición de estos entre el nivel central-central y central-regional, generando con ello duplicidad de actividades que conllevan a presupuestos débiles y por ende a resultados mínimos. (Ver Cuadro 1).

En un país de ricos recursos, como es el caso del Perú, es evidente que los primeros impulsos de desarrollo industrial tienden a manifestarse a través de la generación de valor agregado a esos recursos, pero de hecho, en las últimas dos décadas el Perú ha venido pretendiendo seguir una estrategia de diversificación productiva y de las exportaciones sustentada en la agregación de valor a sus recursos naturales más abundantes, en agro, pesca y minería, pero no lo ha logrado, es recientemente que con la agricultura se puede decir

Cuadro 1. Sobreposición de programas de sectores productivos entre el nivel central y regional al 2010.

\begin{tabular}{|c|c|}
\hline Programas/sector & Actividades/proyectos \\
\hline \multicolumn{2}{|r|}{ Competitividad } \\
\hline MINAG & Promoción de la competitividad Agraria en Piura \\
\hline PRODUCE & Promoción de la competitividad Industrial \\
\hline Gob.Reg.de PIURA & Fortalecimiento de la competitividad \\
\hline \multicolumn{2}{|c|}{ FORTALECIMIENTO DE CADENAS PRODUCTIVAS } \\
\hline PRODUCE & Fortalecimiento de la cadena productiva de óxido de cal en Pasco \\
\hline Gob.Reg. de PASCO & Fortalecimiento de la cadena productiva Industrial \\
\hline PRODUCE & Fortalecimiento de la cadena productiva del palto en Ayacucho \\
\hline Gob.Reg. DE AYACUCHO & Fortalecimiento de la cadena Productiva del Palto \\
\hline MINAG & Fortalecimiento de la cadena productiva del Palto \\
\hline SIERRA EXPORTADORA & Fortalecimiento de la cadena productiva del Palto \\
\hline \multicolumn{2}{|l|}{ GESTIÓN } \\
\hline PRODUCE & Prácticas modernas de gestión empresarial en las MYPEs \\
\hline MINAG & Gestión para el desarrollo del agro \\
\hline PESCA & Apoyo al desarrollo de la gestión empresarial \\
\hline GGRR & Fortalecimiento de las capacidades de gestión Empresarial \\
\hline
\end{tabular}

Fuente: Plan Agrario Sectorial Multianual 2012-2016, Plan Estratégico Sectorial de Energía y Minas Multianual 2008-2011 y Plan Estratégico Sectorial de Producción Multianual 2011-2015. Elaboración propia 
que se está mejorando el valor agregado con las exportaciones no tradicionales. En minería es casi nulo el valor agregado que se les da a los minerales e hidrocarburos.

Especialmente en la presente década, ha habido un despliegue de múltiples esfuerzos en esa orientación por parte del Ministerio de la Producción, el Ministerio de Comercio Exterior y Turismo, el Ministerio de Agricultura, el Ministerio de Trabajo, los gobiernos regionales y locales y diversos organismos vinculados (Consejo Nacional de Competitividad, IMARPE, ITP, Red de CITE, PROMPERÚ, COFIDE, PROINVERSIÓN, CONCYTEC, INDECOPI, etc.). Sin embargo, estos esfuerzos desde el Estado han sido signados por la desarticulación, las duplicidades y la ausencia de una gestión por resultados sustentados en una ingeniería interinstitucional inconsistente. (Ver Cuadro 2).

La falta de políticas integrales de desarrollo de la actividad productiva hace que no exista la normatividad y los instrumentos integrales para impulsar el desarrollo productivo y la competitividad sistémica, esto exige la necesidad de reorganizar la institucionalidad del ente sector de estas actividades (Ministerio de la Producción) y que defina una estructura de la actividad productiva diversificada sostenible e innovadora, de creciente

Cuadro 2. Muestra sobre la dispersión y atomización existentes en dos sectores productivos. Programas financiados al año 2010.

\begin{tabular}{|c|c|c|}
\hline Sector & Sub-sector & Programa \\
\hline \multirow{5}{*}{ AGROPECUARIO } & \multirow[t]{3}{*}{ 1. Agrario } & $\begin{array}{l}\text { 1.1. Asesoramiento y apoyo } \\
\text { 1.2. Innovación tecnológica } \\
\text { 1.3. Eficiencia de mercados }\end{array}$ \\
\hline & & $\begin{array}{l}\text { 1.4 Desarrollo alternativo } \\
\text { 1.5. Promoción del comercio interno } \\
\text { 1.6. Protección sanitaria vegetal } \\
\text { 1.7. Inocuidad agroalimentaria } \\
\text { 1.8. Forestación y reforestación }\end{array}$ \\
\hline & & $\begin{array}{l}\text { 2.1. Innovación tecnológica } \\
\text { 2.2. Asesoramiento y apoyo }\end{array}$ \\
\hline & \multirow{2}{*}{ 2. Pecuario } & $\begin{array}{l}\text { 2.3. Protección sanitaria animal } \\
\text { 2.4. Inocuidad pecuaria }\end{array}$ \\
\hline & & 2.5. Eficiencia de mercados \\
\hline \multirow{4}{*}{ PRODUCCIÓN } & \multirow[b]{3}{*}{ 3. Industria } & 3.1. Asesoramiento y apoyo \\
\hline & & 3.2. Promoción de la Industria \\
\hline & & $\begin{array}{l}\text { 3.3. Innovación tecnológica } \\
\text { 3.4. Eficiencia de mercados } \\
\text { 4.1.Asesoramiento y apoyo } \\
\text { 4.2. Innovación tecnológica }\end{array}$ \\
\hline & 4. Pesca & $\begin{array}{l}\text { 4.3. Regulación y administración del recurso } \\
\text { ictiológico } \\
\text { 4.4. Fomento de la producción } \\
\text { Acuícola } \\
\text { 4.5. Formación ocupacional }\end{array}$ \\
\hline
\end{tabular}

Fuente: Ministerio de Economía y Finanzas (SIAF)al 2010. Elaboración propia 
valor agregado, de calidad, con cadenas y conglomerados competitivos a nivel nacional, regional y local.

La siguiente lámina ilustra algunas de las principales Políticas Sectoriales, en la que se puede apreciar la falta de articulación entre los sectores productivos, al extremo de que el sector minería no tiene absolutamente nada ni entre el mismo ni con los demás. Es en función a esta desarticulación de políticas integrales que la presente investigación propone dentro de las Reformas del Estado la modificación del Sector Producción para que se integren los otros sectores faltantes, tales como el de Agricultura y Energía y Minas.(Ver cuadro 3).

También se pudo apreciar en el Marco Teórico que las actividades productivas se encuentran bajo el ámbito de diferentes Ministerios: Ministerio de la Producción (Industria y Pesquería), Ministerio de Agricultura y Ministerio de Energía y Minas, no existiendo una coherente articulación entre las actividades productivas extractivas con las actividades productivas de transformación; es más cada uno de estos sectores tiene su propia legislación que norma su objetivo, finalidad, ámbito y funciones; además dispone de leyes especificas para las actividades que forman parte de su competencia, no obstante existiendo una norma legal que indica que se deben integrar estos Ministerios en uno solo. En el siguiente cuadro se puede apreciar la articulación productiva (ver cuadro $\mathrm{N}^{\circ} 8$ ), que daría paso a la integración de los sectores productivos bajo un solo Ministerio y en las regiones bajo las gerencias regionales y a nivel local solamente en cuanto al desarrollo empresarial, sin mayores injerencias en las políticas integrales de desarrollo productivo, por no tener funciones al respecto.

Cuadro 3. Políticas públicas de los sectores productivos contenidas en sus planes sectoriales multianuales comprendidas en materia de articulación productiva.

\begin{tabular}{|c|c|c|}
\hline stoger retictures: & pottospass & WITOSEFFCL \\
\hline & 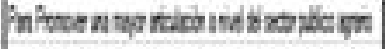 & 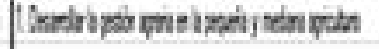 \\
\hline & 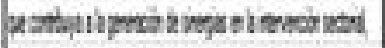 & \\
\hline & 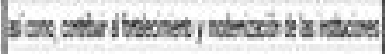 & 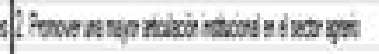 \\
\hline \multirow[t]{3}{*}{ Nater } & 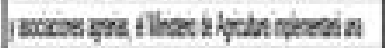 & \\
\hline & 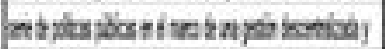 & 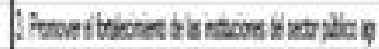 \\
\hline & 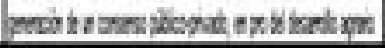 & \\
\hline \multirow[t]{6}{*}{ ng. } & 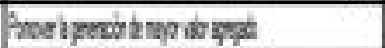 & Destanituscastos \\
\hline & Bizennei & \\
\hline & & 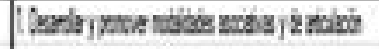 \\
\hline & & 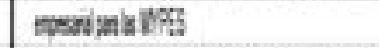 \\
\hline & 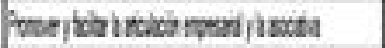 & 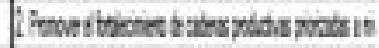 \\
\hline & & 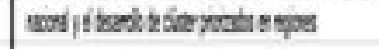 \\
\hline \multirow[t]{8}{*}{ NUSTE } & & 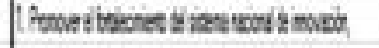 \\
\hline & & 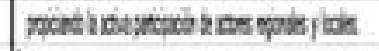 \\
\hline & 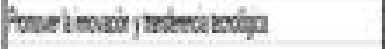 & 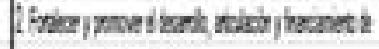 \\
\hline & & ans imairevigh \\
\hline & & 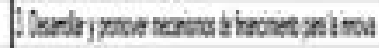 \\
\hline & & 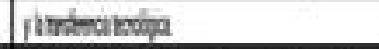 \\
\hline & & 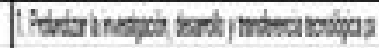 \\
\hline & foreitrank 2 anes 2 abe & 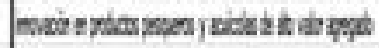 \\
\hline \multirow[t]{3}{*}{ KOUEA } & pim zues yeids & 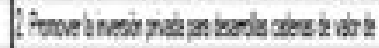 \\
\hline & & materestat \\
\hline & & 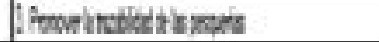 \\
\hline
\end{tabular}

Fuente: Tomado del Plan Agrario Sectorial Multianual 2012-2016, Plan Estratégico Sectorial de Energía y Minas Multianual 2008-2011 y Plan Estratégico Sectorial de Producción Multianual 2011-2015. Elaboración propia. 
Cuadro 8. Articulación productiva.

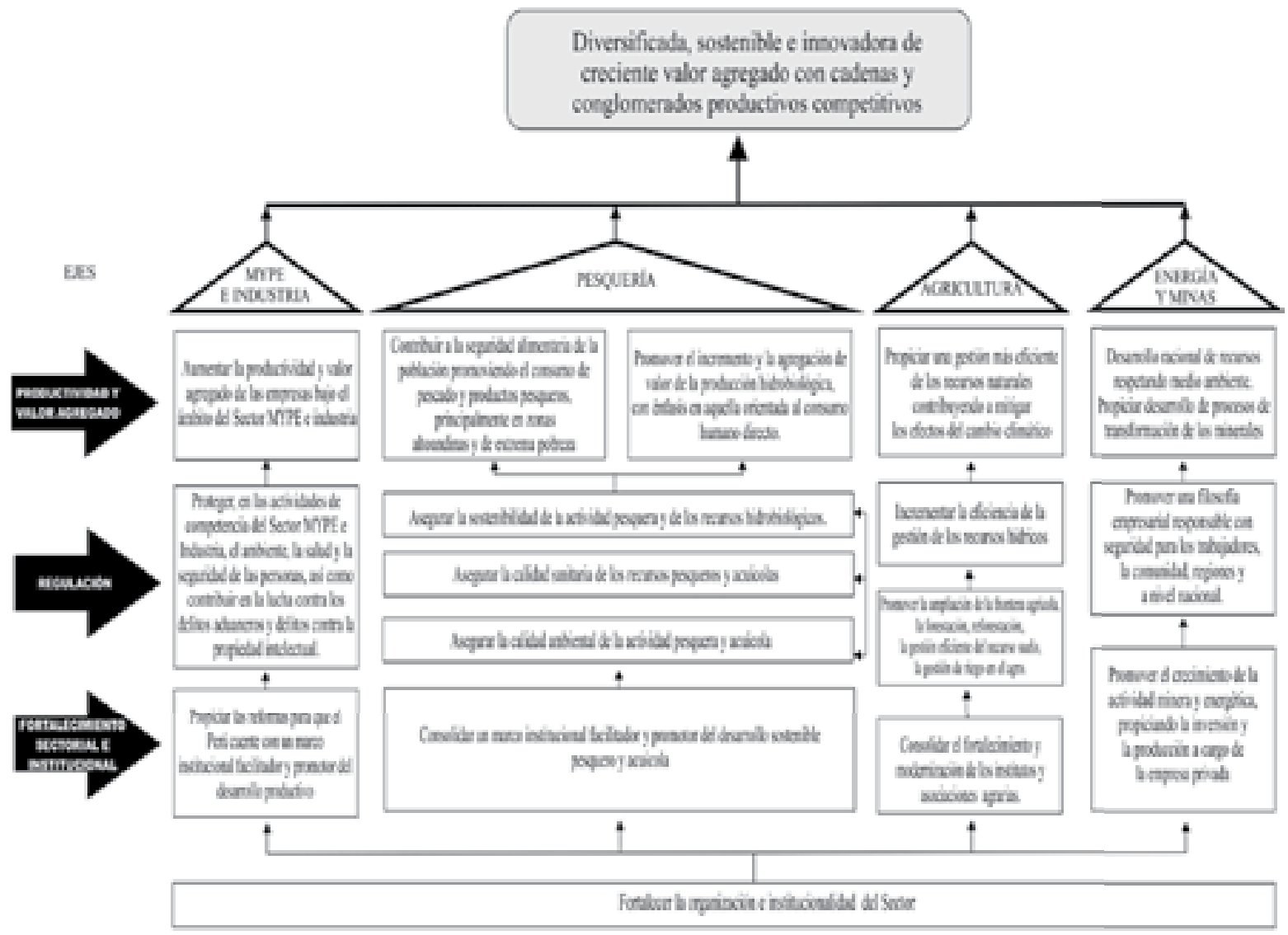

Fuente: Elaboración propia.

A partir de la articulación productiva y dentro del marco de la reforma del Estado se propone la reformulación de la Ley del Ministerio de la Producción integrado por los sectores productivos (Industria, Pesquería, Agricultura y Energía y Minas) como aporte de la investigación al desarrollo integral de las actividades económico-productivas del país.

A nivel regional, también se propone una estructura coherente con la propuesta del nivel central, de tal forma que los sectores no estén sueltos y dependientes del nivel central sino bajo las gerencias regionales como órganos desconcentrados de la región. (Ver cuadro 9)

\section{PROPUESTAS DE POLÍTICAS PÚBLICAS}

1. En resumen y con la finalidad de hacer que funcione el aparato público en apoyo a la actividad empresarial nacional, se propone las siguientes políticas públicas:

2. Política de desarrollo integral y articulado de la actividad productiva nacional para mejorar la innovación y la competitividad.
3. Política de responsabilidad empresarial y de distribución de utilidades acordes con la normatividad vigente.

4. Política de desarrollo de infraestructura para facilitar la comercialización nacional e internacional en las mejores condiciones de operatividad.

5. Política de desarrollo tecnológico y financiero que alcance también a las MYPE

6. Política de intervención del estado a través de la formación de equipos profesionales especializados en apoyo al desarrollo empresarial, articulado a nivel central, regional y local, que promueva el desarrollo socioeconómico del país en su conjunto.

7. Política de capacitación especializada a todos los trabajadores de las empresas tanto públicas como privadas para facilitarles el aprendizaje de las nuevas tendencias que acarrea las tecnologías de punta. 
Cuadro 9. Estructura orgánica del Ministerio de la Producción.

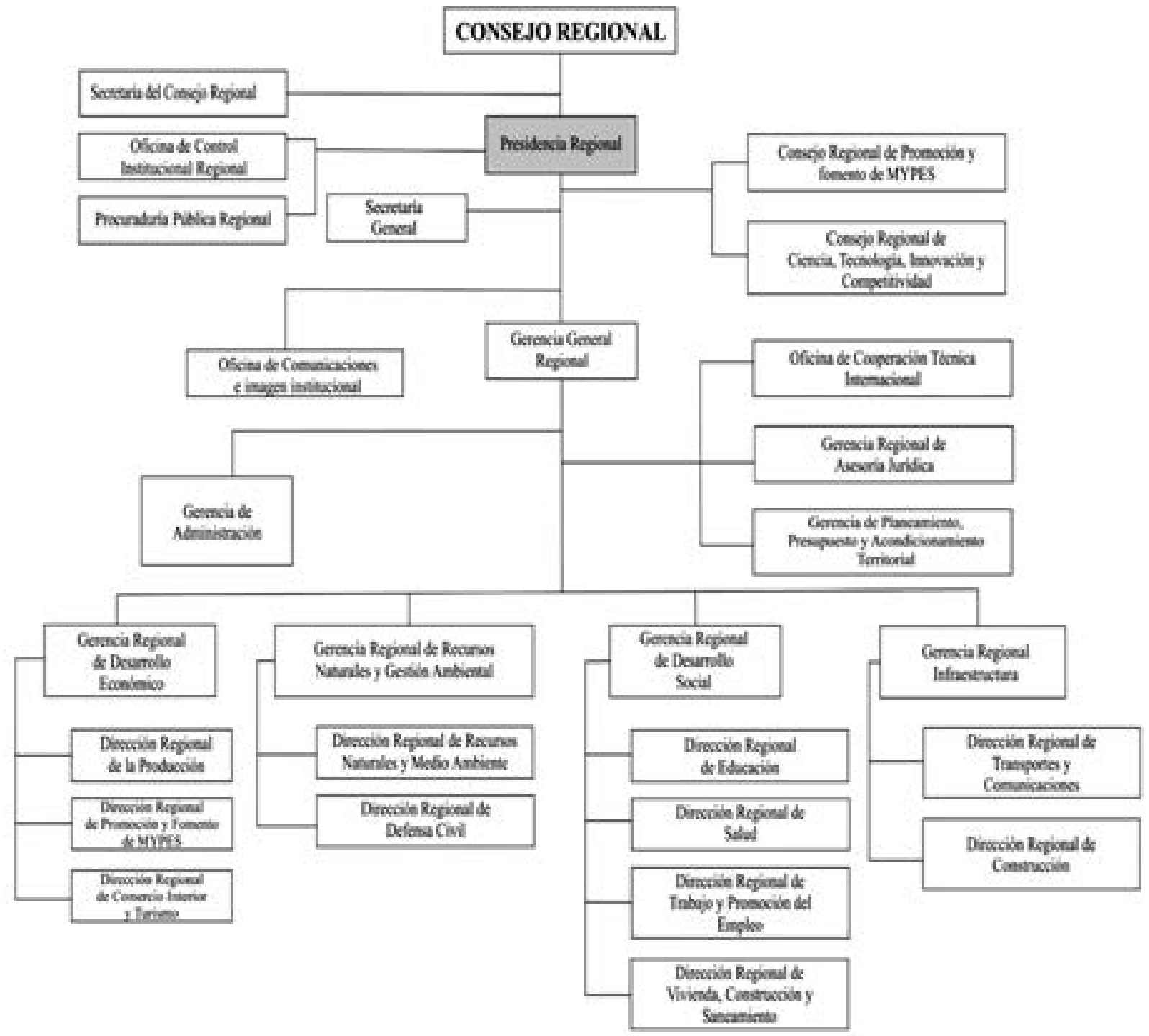

\section{CONCLUSIONES}

1. Los sectores extractivos y de transformación son organismos dependientes del Poder Ejecutivo y a la fecha los esfuerzos realizados no tienen resultados concretos, debido a la ausencia de objetivos claros y precisos de un ordenamiento institucional para el desarrollo integrado y articulado de estos.

2. Los diversos Sectores de la actividad extractiva y de transformación se encuentran bajo la administración y gestión de diferentes Ministerios y cada cual desarrolla sus funciones sin ninguna planificación ni orientación de lo que el país necesita. Este hecho hace que la gestión pública siga una suerte de desorden dentro de un país tan rico en recursos naturales y humanos, pero tan frágil en la definición de prioridades reales que conlleven a no tener claro lo que necesita nuestro país en esta materia, a nivel nacional, regional y local.

3. La falta de un reordenamiento institucional que integre en un solo Organismo a todas los Sectores relacionados con la actividad extractiva y de transformación, por la carencia de políticas integrales de desarrollo de la actividad productiva.

4. En la última década ha habido un despliegue de múltiples esfuerzos para impulsar la generación de valor agregado y contenido tecnológico a nuestros recursos naturales; sin embargo 
Cuadro 10. Estructura orgánica de un Gobierno local.

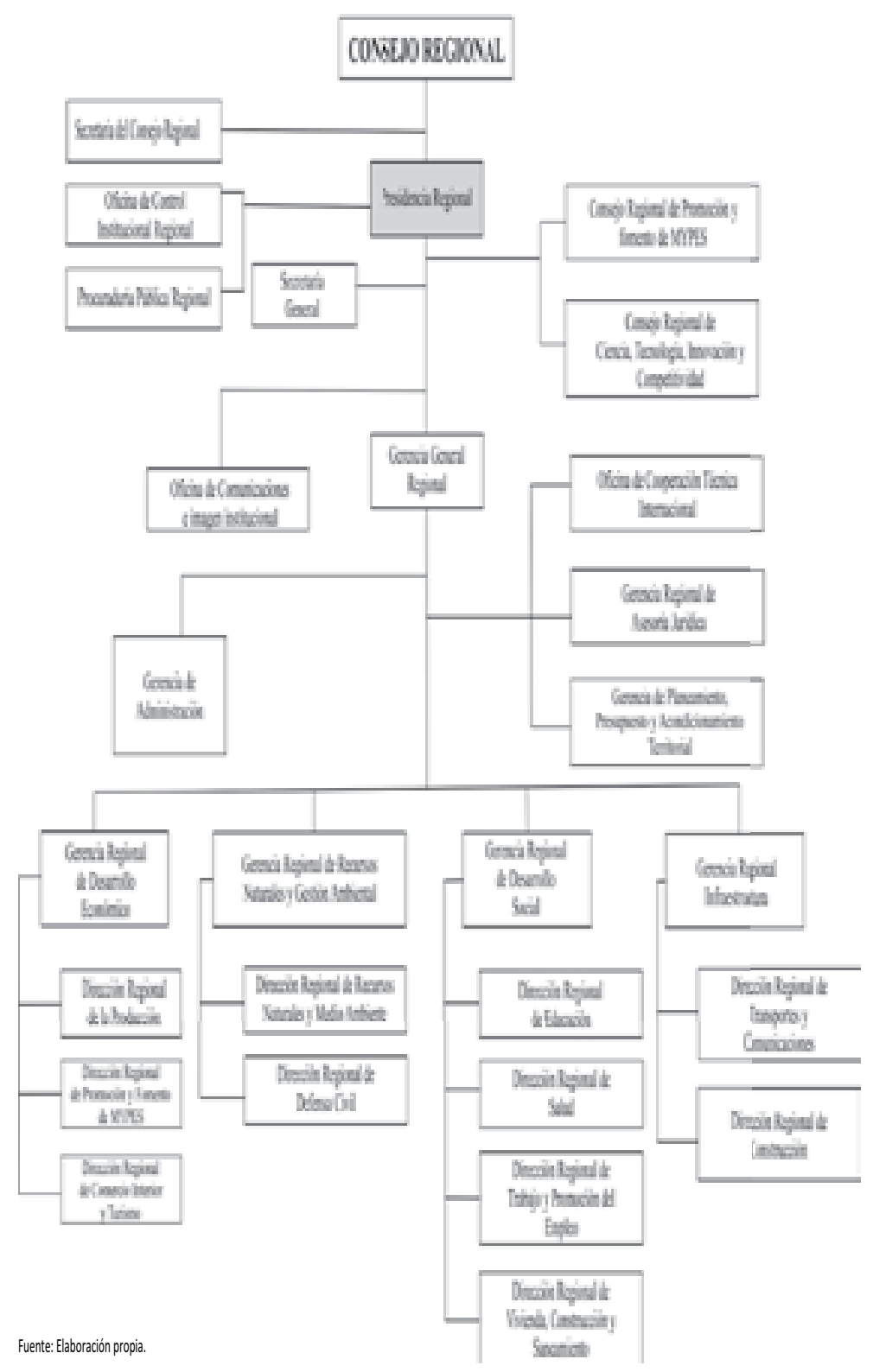

estos esfuerzos han estado signados por la desarticulación, las duplicidades y la ausencia de una política que articule los sectores productivos transformadores de los recursos naturales que generen cadenas productivas.

5. La diversificación productiva es a todas luces un proceso trunco tratándose de un país abundante en recursos naturales y en el tiempo no se ha desarrollado actividades intensivas en conocimiento e innovación tecnológica.

\section{RECOMENDACIONES}

1. Articular todos los sectores productivos involucrados dentro del marco institucional de una Reforma del Estado, que propicie el fortalecimiento Institucional de las actividades extractivas y de transformación bajo el enfoque sistémico para que se formule una política de Estado coherente y un Plan Nacional de la Producción, debidamente coordinado a nivel nacional, regional y local, orientando así las políticas, planes y programas en función del 
desarrollo integral del país en el mediano y largo plazo, para el mejor aprovechamiento de los recursos e infraestructura a nivel micro y macro regional.

2. Que el nuevo Ministerio de la Producción formado por los cuatro sectores productivos, promueva el desarrollo de todas las actividades de extracción y transformación de manera articulada, propiciando las cadenas de valor, de tal forma que se facilite el incremento de la productividad, con la finalidad de poder garantizar en el mediano y largo plazo la competitividad de los bienes y servicios que oferta el país.

3. Contribuir con el actual Gobierno a través de la presente investigación, para que se propicie la Organización de una Administración Pública eficiente y gestión moderna de sus capacidades en el marco de la Reforma del Estado y con la propuesta de políticas públicas integradoras.

4. Que la propuesta proveniente de la presente investigación, dentro del Marco de la Reforma del Estado, el Poder_Legislativo lo haga suyo, lo mejore y perfecciones a fin de modificar el Decreto Legislativo $N^{0} 1047$, Ley de Organización y Funciones del Ministerio de la Producción, incorporando los sectores Agricultura y Energía y Minas, para ello se adjunta en la investigación el Proyecto de Ley que modifica la Ley Orgánica del Ministerio de la Producción y su correspondiente estructura orgánica para los tres niveles (Central, Regional y Local).

\section{REFERENCIAS BIBLIOGRÁFICAS}

[1] Abugattás, L. (2002) "Política Industrial en el Perú: Balance y Márgenes de Acción". En: Fairlie (ed). El sector industrial peruano en el Nuevo Contexto Internacional. Lima: Fundación Ebert.

[2] Kuramoto, J. (2001). "Aglomeraciones mineras en el Perú". En Aglomeraciones mineras y desarrollo en América Latina, CIID.

[3] Medellín T., pedro (1997). "La Política de las Políticas Públicas", propuesta teórica y metodológica, para el estudio de las políticas públicas en países de frágil institucionalidad, CEPAL, Santiago de Chile, 2004.

[4] MINAG (2007). "Plan Estratégico Sectorial Multianual Actualizado del Ministerio de Agricultura 2007-2011", Lima, Agosto de 2010. Unidad de Política Sectorial Oficina de Planeamiento y Presupuesto.

[5] MINEM (2005). "Plan estratégico Institucional del Ministerio de la Producción, 2005-2007", Lima, 2007, Unidad de Política Sectorial Oficina de Planeamiento y Presupuesto.

[6] Oszlak, Oscar, y O’ Donnell (1997). "Políticas Públicas y Regímenes Políticos", visitado en www.ccee.edu.uy/.../.

[7] PRODUCE (2007). "Plan estratégico Institucional del Ministerio de la Producción, 2004-2006", Lima, 2007, Unidad de Política Sectorial-Oficina de Planeamiento y Presupuesto. 